\title{
A rare case of Takayasu's arteritis in pregnancy
}

\section{Sanjay Singh*, Uttara Aiyer Kohli, Manash Biswas, Moman Singh}

Department of Obstetrics \& Gynaecology, Armed Forces Medical College Pune, Maharashtra, India

Received: 19 December 2015

Accepted: 07 January 2016

\author{
*Correspondence: \\ Dr. Sanjay Singh, \\ E-mail: drsanjaysingh@gmail.com
}

Copyright: () the author(s), publisher and licensee Medip Academy. This is an open-access article distributed under the terms of the Creative Commons Attribution Non-Commercial License, which permits unrestricted non-commercial use, distribution, and reproduction in any medium, provided the original work is properly cited.

\begin{abstract}
Takayasu's arteritis (TA) is an uncommon, chronic inflammatory vascular disease of unknown etiology that primarily involves aorta and its branches and pulmonary arteries. Though it is present worldwide, it is more prevalent in Asian countries. It usually presents during reproductive age group with non-specific symptoms. The symptoms of the disease are varied and patients can present asymptomatically with impalpable pulses. There are no specific laboratory tests to diagnose TA. Angiographic imaging is considered to be the gold standard investigation in diagnosing TA. Steroids with subsequent tapering doses are the mainstay of medical treatment. Reconstructive vascular surgery is limited to the severe and irreversible stenotic lesions. Though the disease process theoretically is not aggravated by the pregnancy, development of preeclampsia and fetal growth restriction may occur during pregnancy. Preconception counseling and careful monitoring of pregnant mother by a multidisciplinary team, improves the pregnancy outcome. Here we present a bad obstetric case with TA who subsequently developed severe preeclampsia and fetal growth restriction. The pregnancy outcome could be made favourable by strict monitoring and multidisciplinary team approach.
\end{abstract}

Keywords: Takayasu's arteritis, Pregnancy, Preeclampsia, Fetal growth restriction

\section{INTRODUCTION}

Takayasu's arteritis (TA) is a chronic inflammatory vascular disease of idiopathic etiology which primarily involves aorta and its branches and pulmonary arteries. ${ }^{1,2}$ It is seen predominantly in young women of reproductive age group and is more prevalent in Asian countries. ${ }^{3}$ It usually presents with non-specific symptoms and there are no specific laboratory tests to diagnose it. The gold standard investigation to diagnose TA is angiographic imaging. ${ }^{4}$ Whereas, steroids with tapering doses are the mainstay of medical treatment; the refractory cases require additional therapy. ${ }^{5}$ Reconstructive vascular surgery is limited to the severe and irreversible stenotic lesions. ${ }^{3}$ Here we describe the course and management of pregnancy in a woman with Takayasu's arteritis.

\section{CASE REPORT}

25 years old G6P2L0A3 lady a case of bad obstetric history reported in the first trimester of pregnancy for routine antenatal care. Her first two pregnancies were intrauterine fetal death due to abruption at 34 weeks and 30 weeks respectively. Her third, fourth and fifth pregnancies were mid trimester missed abortions at 18 , 20 and 21 weeks respectively. Her last menstrual period was 17 February 2015 making the expected date of delivery 24 November 15 . She was diagnosed to have central nervous system inflammatory granuloma induced seizure disorder in 2007 for which she was put on antiepileptic medication that continued for three years during which she remained seizure free. On clinical examination she was detected to have absent radial and brachial pulses both upper limbs with hypertension (blood pressure $-150 / 100 \mathrm{mmHg}$ ) and tachycardia (pulse rate- $120 / \mathrm{min}$ ) measured in lower limbs. 
Blood test for renal (urea \& creatinine), liver and thyroid function tests, anti-nuclear antibody, anti-phospholipid antibodies and thrombophilia profile were normal. However, she had microcytic hypochromic anemia $(\mathrm{Hb} \%$ - 8.4 gm \%). She also developed significant proteinuria (24 hour urinary protein $2316 \mathrm{mg} / \mathrm{dl}$ ) at 20 weeks of pregnancy. Echocardiography suggested normal study. Ultrasonography and Doppler study of the kidneys, adrenals and renal vessels were normal. Her MRI brain showed calcified inflammatory granulomas in the left precentral and right middle frontal gyrus and few discrete hyperintense white matter lesions. EEG was normal. MRI of Thoracic Aorta showed luminal narrowing with asymmetric aortic wall irregularity of the lower thoracic and abdominal aorta which was seen to progress craniocaudal with maximum stenosis (aortic diameter 8.3 $\mathrm{mm})$ at the level of crossing of left renal vein. Findings were suggestive of aorta arteritis- Takayasu's arteritis type III (Figure 1, 2 and 3). Doppler study of both upper limbs was suggestive of bilateral proximal stenotic occlusive disease - subclavian arteritis.

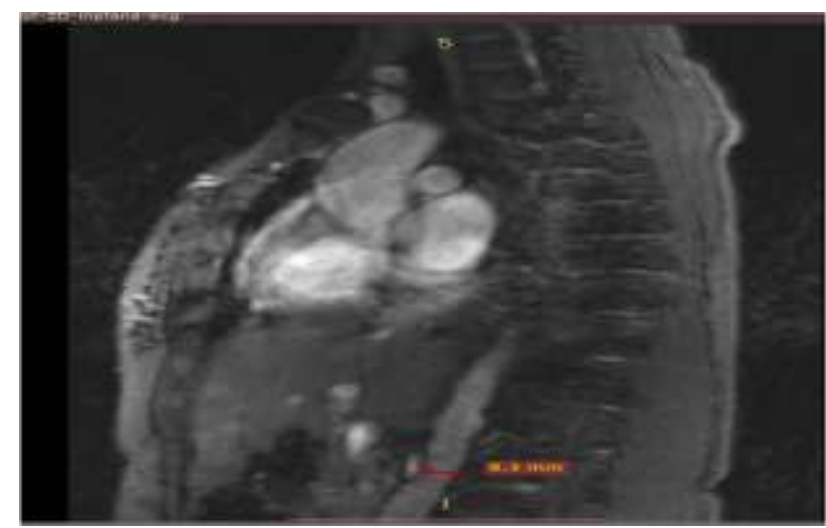

Figure 1: 2D Time of flight (TOF) sagittal image reveals luminal narrowing of abdominal aorta with narrowest diameter measuring $8.3 \mathrm{~mm}$.

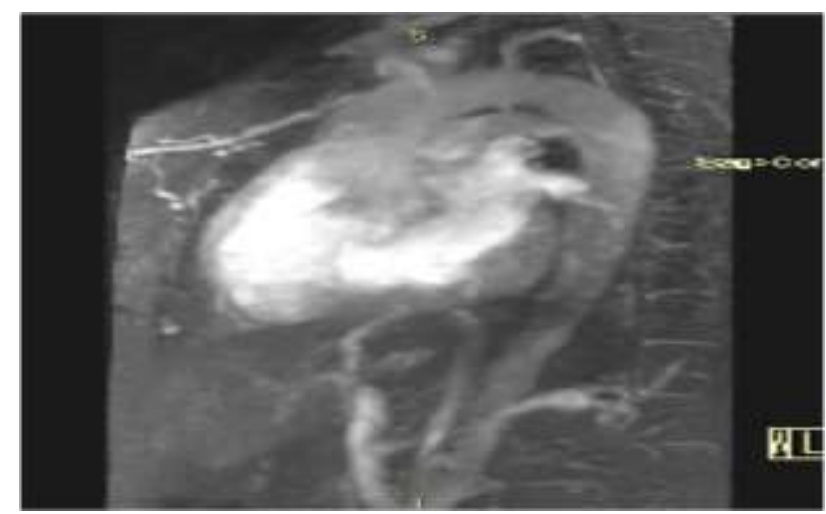

Figure 2: Maximum intensity projection (MIP) image shows lower thoracic and abdominal aorta wall irregularity and luminal narrowing.

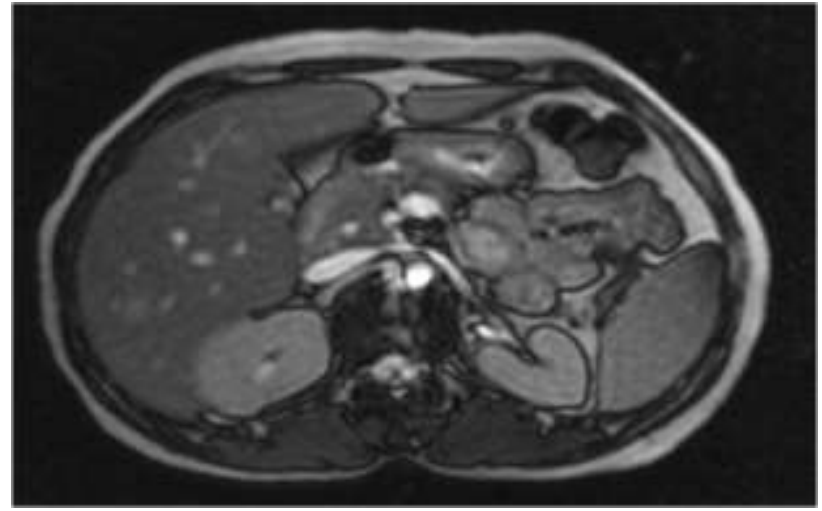

Figure 3: True fast imaging with steady state precision (FISP) axial section at the level of renal veins shows luminal narrowing of the aorta.

She was diagnosed as a case of Takayasu's aorta arteritis with chronic hypertension with bad obstetric history $(\mathrm{BOH})$. She in due course of time developed superimposed pre eclampsia too. She was jointly managed by the Obstetrician, Rheumatologist and Cardiologist. She was initially put on prednisolone $40 \mathrm{mg}$ daily for first four weeks, which was gradually tapered to $10 \mathrm{mg}$ per day over another 4 weeks. She was also put on ecosprin $75 \mathrm{mg}$ and low molecular weight heparin $40 \mathrm{mg}$ sub cutaneous from early pregnancy. She was initially put on metoprolol $25 \mathrm{mg}$ twice a day along with amlodipine $10 \mathrm{mg}$ once daily for the control of blood pressure (BP) and tachycardia. However her BP kept on rising (highest recorded BP-190/118 $\mathrm{mmHg}$ in the lower limbs), for which further antihypertensive in form of gradual increasing doses of labetalol (200 mg thrice daily) and alpha methyl dopa (500 $\mathrm{mg}$ four times daily) had to be added. Metoprolol was stopped once labetalol was added. BP finally was controlled between 160/100 in lower limb and 140/90 in right upper limb on these three antihypertensive. From mid trimester onwards she was followed up with regular haemogram, liver and renal function tests and sonography for fetal growth and umbilical artery Doppler for assessment of fetal wellbeing. Her condition remained stable and she was given steroids for enhancing fetal lung maturity at 27 weeks in view of risk of preterm delivery. She developed oligohydramnios so ecosprin was stopped and at 34 weeks 6 days and was delivered by caesarean section in view of growth restriction with absent umbilical artery diastolic flow. She delivered a $1.87 \mathrm{~kg}$ female baby with normal APGAR. Her intraoperative and postoperative period was uneventful and she was discharged once the baby showed a normal weight gain (1950 gms on discharge) and her BP got settled. On discharge her BP was $110 / 86$ in the UL and 140/80 in the lower limb. She was on tab amlodipine 10mg BD and prednisolone $5 \mathrm{mg}$ OD.

The most difficult part of management of this patient was controlling her blood pressure and continuation of pregnancy inspite of significant proteinuria. The risk of 
maternal and foetal complications including abruption and IUD was present throughout and strict monitoring for maternal and fetal wellbeing and inpatient management of the pregnancy helped in a positive outcome.

\section{DISCUSSION}

Takayasu's disease was first reported in 1908 by two Japanese ophthalmologists, Takayasu's and Onishi, who observed retinopathy occurring with absent limb pulses. ${ }^{6}$ As can be seen in our case, it occurs predominantly in young women, (female: male ratio 8:1) of reproductive age group. TA has world-wide distribution, but is more prevalent in Asian populations. ${ }^{3}$ This rare entity with an incidence of 1-2 per million is a chronic inflammatory vascular disease of idiopathic etiology principally affecting major vessels such as the aorta, its branches and the pulmonary arteries. ${ }^{1,7}$ Four types of Takayasu arteritis can be identified: Type I (disease involving the aortic arch and its branches), type II (lesions restricted to descending thoracic aorta and abdominal aorta), type III (patients have characteristics of types I and II), and type IV (involvement of the pulmonary artery). ${ }^{8}$ Our patient had Type-III involvement.

Classically the disease may have a triphasic course. In the initial active phase of the disease patient presents with vague constitutional symptoms such as malaise, weight loss, fever, myalgias and arthralgias, as a result of which diagnosis may be missed as can be noted in our case. This phase is generally associated with increase in C-reactive protein and or erythrocyte sedimentation rate i.e. acute phase reactants. This progresses to a second phase with symptoms of cerebral, visceral or extremity ischemia. This in due course culminates in a final 'burnt-out' quiescent phase of fibrosis. The severity and progression of vessel involvement are extremely variable and these 'typical' symptoms are seen in only 33 per cent of all patients. ${ }^{4}$ The clinical diagnosis of Takayasu's arteritis as per the American College of Rheumatology, 1990 requires the presence of three or more of the following six criteria's : onset of age less than or equal to 40 years, claudication of an extremity, decreased brachial artery pulse, greater than $10 \mathrm{mmHg}$ difference in systolic blood pressure between arms, a bruit over subclavian arteries or the aorta and arteriographic evidence of narrowing or occlusion of the entire aorta, its primary branches, or large arteries in the proximal upper or lower extremities. Four features out of six were present in our case. Diagnosis is largely based on clinical manifestations and angiographic findings like coarctation, occlusion and aneurysmal dilatations.

As per European League Against Rheumatism (EULAR), initial high doses of glucocorticoids is used for the remission of the disease and an immunosuppressive agent is used as an adjunctive therapy. ${ }^{5}$ EULAR recommends the starting dose of glucocorticoids $1 \mathrm{mg} / \mathrm{kg}$ body weight for four weeks and then to taper them off. ${ }^{5}$ We followed the same protocol in our case. Reconstructive vascular surgery is indicated in cases with critical renovascular hypertension, severe claudication, and critical stenosis of cerebral vessels, ischemic crises and moderate aortic regurgitation. ${ }^{3}$

TA follows remitting and relapsing course along with chronic therapy of glucocorticoids. Five year survival has been reported as $88-90 \% .^{9}$ The disease can also be classified into stages according to the presence of major complications such as hypertension, retinopathy, aneurysms, and aortic regurgitation. ${ }^{2}$ Our patient had only hypertension. Death in these patients is mainly due to ischemia, congestive cardiac failure and stroke. ${ }^{10}$

Even though, fertility is not adversely affected and pregnancy does not appear to exacerbate the disease, pregnancy should be considered in remission period. ${ }^{11}$ Hypertensive complications such as preeclampsia and exacerbation of chronic hypertension, and fetal complications such a restriction of intrauterine growth, abortion, and fetal death have been reported in $60 \%$ to $90 \%$ of the cases. ${ }^{11}$ All these complications could be noted in our patient. The high incidence of restricted uterine growth seems to be related to uncontrolled hypertension and aorta and iliac arteries involvement. ${ }^{12}$ Arterial ultrasound Doppler, is useful modality of evaluating fetal well-being and growth in patients with TA and our case certifies this fact.

Preconceptional counselling is important for assessment of severity of the disease and enabling appropriate adjustments or stoppage of cytotoxic drugs prior to conception. All the women should be booked as early as possible. Antenatal care is best provided by a multidisciplinary team as we did that includes an obstetrician, radiologist, rheumatologist, cardiologist, haematologist and anaesthesiologist. ${ }^{13}$ Pregnancy can continue till term and induction of labour is considered in the presence of superimposed pre eclampsia or hypertension. We terminated the pregnancy in view of absent umbilical artery diastolic flow. Elective Caesarean at term is indicated for severe disease e.g. retinopathy, arterial aneurysms, and aortic regurgitation. ${ }^{11}$ Usual doses of dexamethasone or betamethasone for foetal lung maturity is recommended for cases with risk of preterm delivery, as we did in our case.

During post-partum period, progesterone only pill or depot injections or intra uterine devices can be safely recommended. Combined contraceptive pill however is generally avoided as there is increased theoretical risk of thromboembolism. Thromboprophylaxis is recommended in patients with associated anti phospholipids antibodies (aPL). ${ }^{13}$

\section{CONCLUSIONS}

Management of pregnancy with Takayasu disease especially in a complicated variety is a challenge. The disease process though does not impair the fertility and 
does not appear to get aggravated by pregnancy; development of maternal and fetal complications may occur. Preconception counselling is of paramount importance and conception during remission phase is ideal. For optimal pregnancy outcome multidisciplinary management is essential. Adequate control of blood pressure and careful foetal monitoring improves the outcome.

\section{Funding: No funding sources}

Conflict of interest: None declared

Ethical approval: Not required

\section{REFERENCES}

1. Vidhate M, Garg RK, Yadav R, Kohli N, Naphade P, Anuradha HK. An unusual case of Takayasu's arteritis: Evaluation by CT angiography. Ann Indian Acad Neurol. 2011;14(4):304-6.

2. Ishikawa K. Natural history and classification of occlusive thromboaortopathy (Takayasu's disease). Circulation. 1978;57:27-35.

3. Walker BR, Colledge NR, Ralston SH, Penman ID. Davidson's principles \& practice of medicine. 22th ed. India: Elsevier; 2014.

4. Sise MJ, Counihan CM, Shackford SR, Rowley WR. The clinical spectrum of Takayasu's arteritis. Surgery. 1988;104:905-10.

5. Mukhtyar C, Guillevin L, Cid MC, Dasgupta B, de Groot K, Gross W, et al. EULAR recommendations for the management of large vessel vasculitis. Ann Rheum Dis. 2009;68:318-23.
6. Takayasu M. A case with peculiar changes of the retinal central vessels (in Japanese). Acta Soc Ophthal Jpn. 1908;12:554-5.

7. Watts R, Al-Taiar A, Mooney J, Scott D, Macgregor A. The epidemiology of Takayasu arteritis in the UK. Rheumatol. 2009;48(8):1008-11.

8. Lupi-Herrera E, Sanchez-Torres G, Marcushamer J, Mispireta J, Horwitz S, Vela JE. Takayasu's arteritis. Clinical study of 107 cases. Am Heart J. 1977;93(1):94-103.

9. Saab F, Giugliano RP, Giugliano GR. Takayasu arteritis in a young woman: a 4-year case history. Tex Heart Inst J. 2009;36(5):470-4.

10. Kerr GS, Hallahan CW, Giordano J, Leavitt RY, Fauci AS, Rottem M, et al. Takayasu arteritis. Ann Intern Med. 1994;120:919-29.

11. Matsumura A, Moriwaki R, Numano F. Pregnancy in Takayasu arteritis from the view of internal medicine. Heart Vessels. 1992;7(Suppl):120-4.

12. Mahmood T, Dewart PJ, Ralston AJ, Elstein M. Three successive pregnancies in a patient with Takayasu's arteritis. J Obstet Gynaecol. 1997;17:524.

13. Vitthala S, Misra P. Takayasu's Arteritis \& Pregnancy: A Review. The Internet Journal of Gynecology and Obstetrics. 2007;9(2).

Cite this article as: Singh S, Kohli UA, Biswas M, Singh M. A rare case of Takayasu's arteritis in pregnancy. Int J Reprod Contracept Obstet Gynecol 2016;5:552-5. 